\title{
Laboreal
}

Volume $14 \mathrm{~N}^{\circ} 1$ | 2018

O regresso ao emprego após um acidente de trabalho

\section{El enfoque ergológico para una otra evaluación del trabajo}

A abordagem ergológica para uma outra avaliação do trabalho social La démarche ergologique pour une autre évaluation du travail social

The ergology approach to reach a different assessment of the social work

\section{Ingrid Fouchecourt-Dromard}

Traductor. Cécile Zedde-Vallat y María Noel Close

\section{(2) OpenEdition \\ Journals}

Edición electrónica

URL: http://journals.openedition.org/laboreal/538

DOI: $10.4000 /$ laboreal.538

ISSN: 1646-5237

Editor

Universidade do Porto

\section{Referencia electrónica}

Ingrid Fouchecourt-Dromard, «El enfoque ergológico para una otra evaluación del trabajo », Laboreal [En línea], Volume $14 \mathrm{~N}^{0} 1$ | 2018, Publicado el 01 julio 2018, consultado el 23 septiembre 2020. URL http://journals.openedition.org/laboreal/538 ; DOl : https://doi.org/10.4000/laboreal.538

Este documento fue generado automáticamente el 23 septiembre 2020.

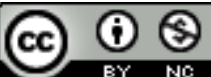

Laboreal está licenciado com uma Licença Creative Commons - Atribuição-NãoComercial 4.0 Internacional. 


\title{
El enfoque ergológico para una otra evaluación del trabajo
}

\author{
A abordagem ergológica para uma outra avaliação do trabalho social \\ La démarche ergologique pour une autre évaluation du travail social \\ The ergology approach to reach a different assessment of the social work \\ Ingrid Fouchecourt-Dromard \\ Tradución : Cécile Zedde-Vallat y María Noel Close
}

\section{REFERENCIA}

Fouchecourt-Dromard, I. (2017). Les Groupes de Rencontres du Travail pour une autre évaluation du travail social. A partir d'une expérience réalisée à la Caisse d'Allocations Familiales des Bouches du Rhône. Thèse de doctorat en philosophie. Aix Marseille Université. France.

\section{NOTA DEL EDITOR}

http://dx.doi.org/10.15667/laborealxiv0118ides

Manuscrito recibido en: enero/2018

Aceptado tras peritaje: mayo/2018

Jurado de tesis : Presidente: Yves Schwartz ; Director de tesis: Renato Di Ruzza; Jurados:

Ewa Bogalska-Martin, Louis Durrive; Examinador: Jean Luc Prades

El análisis siguiente [1] presenta una movilización posible del enfoque ergológico en respuesta a una demanda institucional [ $\left.{ }^{2}\right]$ sobre el tema de la evaluación cualitativa del trabajo social. En introducción y para precisar nuestra problemática, haremos primero, un trabajo de definición.

Es posible concebir el trabajo social en relación con las diferencias que lo oponen a la asistencia y a la caridad cuya misión común es ayudar a las personas, socorrerlas. El 
socorro necesita la inmediatez. Muy a menudo es fáctico, una ayuda financiera o hospitalaria que responde a una necesidad vital, por lo menos urgente. El trabajo social va más allá porque en este caso, solo socorrer es insuficiente. Más allá de la satisfacción inmediata, la persona a la que se ayuda, por un tiempo vulneralizada, debe ser capaz de recuperar su facultad de responder lo mejor posible a las exigencias del mundo que la rodea. Contrariamente a la asistencia y a la caridad, el objetivo del trabajo social, como cualquier actividad curativa (Worms, 2012), no solo es prestar socorro en lo inmediato sino también seguir este socorro gracias al apoyo y al acompañamiento. Al fin y al cabo, el trabajo social resulta ser la combinación de tres contribuciones. La del Estado que propone recursos, dispositivos, socorros; la del trabajador social que se implica personalmente frente a una demanda singular, se esmera en establecer una relación de confianza con otro para acompañarle: ir adónde va el, contribuyendo a sus esfuerzos; en tercer lugar, la persona que solicita, que está comprometida con lo que surge de ella, sus capacidades, sus experiencias, sus habilidades.

La evaluación es un instrumento de medida, un nuevo modelo para ejercer el poder que aparece a finales de los años 1970. Consiste en gobernar por el resultado, gracias a la medida de la ejecución de objetivos cifrados, gracias a indicadores de rendimiento (Ogien in Bocquet, 2016). Esta modalidad es característica de la sociedad moderna haciendo de esta, una técnica de gestión. Cuando se debe evaluar un trabajo que se dirige a seres humanos, varios valores coexisten formando, por lo tanto, dos 'mundos de valores' (Schwartz \& Durrive, 2009a): el de los valores dimensionados con cifras, cantidades que para la mayoría se encuentran en el mundo mercantil; el de los valores no dimensionados que pertenecen a lo político, el 'bien común' (la salud, la educación, les servicios sociales); no existen para estos, limites, o jerarquía entre ellos. Si estos dos mundos coexisten y se mezclan en el dominio social, con utilizar la formulación 'evaluación del trabajo social' se arriesga que no se tenga en cuenta sus posibles contradicciones.

El trabajo social no es una excepción: debe tener en cuenta la regla de las cifras así como también su valor político, tiene que ser medible y rentable. Pero, que es lo que se debe evaluar en el trabajo social: ¿su utilidad social o su coste de producción? ¿El medio o la finalidad? Las evaluaciones que se están haciendo sobre el trabajo social lo descomponen, lo cuantifican y lo traducen en cifras. Tratan más de dimensionamiento (tiempo de trabajo, productividad), o de la satisfacción de los que benefician. Nunca tienen en cuenta lo que sienten los que acompañan. Aunque los conocimientos digitales obtenidos por esas evaluaciones clásicas presenten ciertos aspectos del trabajo de los trabajadores sociales, qué importancia se da a los conocimientos cualitativos, a los que tratan la calidad, de las maneras de practicar, conocimientos que solo puede estar contado, porque son conocimientos personal y propios a cada uno? ¿Si solo se evalúa el socorro, no se distorsiona el juicio de valores?

Por lo tanto, nos formulamos una primera hipótesis: no se puede simplemente evaluar el trabajo social a través de un modelo evaluativo único, un modelo cuantitativo, porque el proyecto político llevado a cabo por el trabajo social no puede traducirse en cifras. Hay que tener en cuenta el sentido del propósito de la acción y las condiciones de su efectividad.

Según la demanda institucional el nuevo modelo evaluativo no debía sustituir a los otros, sino que tendría que complementarlos para que los encargados de tomar decisiones tengan una lectura complementaria. De hecho, la exclusividad de los puntos 
de vista obtenidos hace que su combinación sea obligatoriamente necesaria para presentar de manera exhaustiva la implementación del trabajo social institucionalizado.

La complementariedad es fundamental porque sugiere lo conmensurable, es decir, permite que diferentes entidades evaluadoras sean consideradas de manera igualitaria permitiendo su comparación, su confrontación, y por lo tanto la producción de nuevos conocimientos. La complementariedad invita al diálogo, a la instrucción mutua, al compromiso dialéctico, y fomenta el 'espiral permanente del mejoramiento de los conocimientos' (Schwartz \& Durrive, 2009b, p. 264). De ahí emerge nuestra segunda hipótesis: dando a conocer una visión de los conocimientos que proceden del trabajo real, la evaluación producida puede permitir la transformación de las actividades de trabajo.

Por lo tanto, tuvimos que pensar en formalizar una evaluación original construida a partir de los conocimientos de quiénes trabajan. Es así que implementamos un medio para recoger dichos conocimientos transformando 'esos conocimientos narrativos' (Gori, 2015) en conocimientos conmensurables a los ya producidos, conocimientos teóricos, digitales, que proceden de otras formas de evaluación.

Presentaremos en primer lugar las etapas principales de la evaluación cualitativa que hemos propuesto, y luego intentaremos demostrar cómo esta evaluación puede ser un instrumento interesante como modalidad de management original, especialmente en las Instituciones públicas.

\section{Las grandes etapas de la evaluación ergológica}

Para llevar a cabo esta evaluación, hemos utilizado el enfoque ergológico, que aparece en Francia a principios de los 80 bajo la investigación de Yves Schwartz, filósofo e investigador de la Universidad de Aix-Marseille. Lo central de este enfoque reposa en el concepto de actividad, que se entiende aquí como uso de sí mismo (Schwartz, 2000). Hacer uso de uno mismo es lidiar subjetivamente con los acontecimientos que le suceden a uno mismo. Es hacer uso de su ser físico, biológico, psíquico, social. La actividad es singular, es una animación-negociación personal, un debate personalizado por su propia historia de vida (Schwartz, 2000). Es, como tal, la matriz del conocimiento original e inédito.

La distancia entre el trabajo prescrito y el trabajo real se basa en el punto de encuentro entre prescripciones y una personalidad singular. Esta distancia es el resultado de la actividad del trabajador, del debate que existe entre lo que se exige de sí mismo y lo que otros le piden: la jerarquía, los colegas, los destinatarios del trabajo, etc. Esta distancia concede así a todas las actividades de la actividad humana, su impredecibilidad, su desequilibrio, su variabilidad y, por lo tanto, la precariedad de lo que las define.

Sólo la persona en cuestión puede testimoniar las razones y opciones que explican estas distancias. Es por eso que nada serio puede decirse del trabajo independientemente de quiénes trabajan. Por lo tanto, lo que sienten y sus explicaciones constituyen el punto de vista que el enfoque ergológico [3] quiere obtener y valorizar. Los principios epistemológicos de este se basan en la producción de conocimientos y un principio ético poniendo de relieve la importancia del juicio personal. 
Para realizar la evaluación cualitativa, ergológica, nos hemos necesariamente desviado de las evaluaciones convencionales. A modo de ejemplo, la definición de los indicadores nos pareció que tenían que hacerlo los trabajadores y no quién evaluaba. Fueron los trabajadores los que tuvieron que indicar lo que produce valor para ellos en la realización de su trabajo. Un paso de lado también acentuado por el hecho de que el enfoque al que nos referimos no sigue los procedimientos tradicionalmente aceptados en las ciencias humanas y sociales (no se trata de discutir sobre los hechos o aprobar hipótesis a través de experimentos empíricos. Es más bien acompañar la confrontación, a un nivel igualitario, del conocimiento digital y el conocimiento derivado de la experiencia para que ambos puedan ser fructíferos.

Ya que hemos considerado que el que hace es más capacitado para evaluar, decidimos promover el fomento del conocimiento derivado de la actividad e identificar en los intercambios, los valores a partir de los cuales cada uno analiza las diferencias entre lo prescrito y lo real, de identificar las recurrencias en la forma en que realizan su trabajo, es decir, actualizar lo que se hace para ser eficaz y porque lo hacen, individual y colectivamente. Dicho esto, este trabajo evaluativo no consiste en la realización de un catálogo de conocimientos singulares. No tuvimos que identificar lo que se relacionaba exclusivamente con uno mismo, sino más bien, lo común en el colectivo. Tampoco tuvimos que presentar las diferentes formas de enfoques de colaboración informal, sino que tratamos de entender lo que favorece su despliegue o, al contrario, lo que lo inhibe para permitir que cada uno interrogue sus formas de hacer las cosas con respecto a los nuevos conocimientos que se le presentan a él.

Como las diversas formas de evaluación que existen en la institución, debían complementarse, los conocimientos narrativos debían alcanzar el mismo nivel de importancia científica que el conocimiento digital. Lo que significa que los conocimientos derivados de las experiencias tenían que ser conceptualizados. La conceptualización implica el control de la sistematicidad. Como resultado, el acceso al trabajo real es posible a partir de los conocimientos producidos singularmente en sus convergencias colectivas (sistematicidad). Los puntos de acuerdo sobre el significado dado al trabajo permiten la transición a la abstracción necesaria para el trabajo de conceptualización.

El paso hacia la conceptualización fue posible gracias a un dispositivo original propuesto por el enfoque ergológico, que son los 'Groupes de Rencontres du Travail (GRT)' ('Grupos de Encuentros del Trabajo'), grupos de análisis de la experiencia (Durrive, 2015), donde se invita a los profesionales a reflexionar y discutir sobre su trabajo. En los GRT, la producción de los conocimientos se basa en la dinámica entre, por una parte, los conocimientos teóricos (producción anticipada de los conocimientos sobre la actividad del trabajo: formaciones, prescripciones, reglas, descripción de puesto de trabajo, etc.) y por otra, los conocimientos derivados de la actividad, de la realidad de los trabajadores. La dinámica entre estas dos extremidades implica un beneficio mutuo si se mantiene en un diálogo que acepta que estas formas de conocimientos [4] tienen la misma importancia. Esto significa que estos conocimientos se consideran con la misma importancia permitiendo su confrontación.

Hemos optado por considerar la efectividad del trabajo social como un 'problema común' que se articula entre la actividad (movilización de sí mismo), la organización y lo político. En referencia a algunos aspectos metodológicos del socio-psicoanálisis institucional [5], se imaginó así la implementación de un diálogo igualitario 
tridimensional: entre compañeros (cuatro profesiones fueron solicitadas), entre colegas-compañeros de equipo (estas cuatro profesiones forman el equipo de las intervenciones del trabajo social) y entre los operadores y los desarrolladores de proyectos (agentes de dirección).

La evaluación propuesta se construyó en tres etapas: acompañar la enunciación de los conocimientos personales, acompañar la construcción de puntos de vista compartidos, y finalmente establecer la conmensurabilidad entre las evaluaciones. Se convierte así en una evaluación construida a partir de dos evaluaciones que se basan en las siguientes preguntas: ¿Cuál es mi trabajo? ¿Cómo se hace trabajo social juntos? Se utilizaron los GRT para construir estas dos evaluaciones. Estos grupos se desarrollaron en 9 sesiones de 3 horas, una vez al mes, durante quince meses. Los GRT están hechos de tres fases: aprehender la actividad; construir una perspectiva colectiva sobre el trabajo entre colegas; entender el trabajo colectivo, el trabajo en equipo.

La primera fase del GRT corresponde a la identificación de conocimientos y facilitar el intercambio de experiencias personales entre colegas. Para ello se han movilizado otras técnicas cualitativas para recabar los: observación y entrevista libre. El esfuerzo de adhesión a las situaciones era por lo tanto necesario, en esta primera etapa, para desmitificar las evidencias y profundizar las apariencias. Después de cada sesión, se hizo una síntesis de los distintos elementos de conocimientos obtenidos. Esta síntesis se distribuyó a los participantes antes del encuentro siguiente para aprobarla. También fue la oportunidad para los profesionales de confrontarse, una vez más, a sus conocimientos, enunciados en el mes anterior.

La segunda fase del GRT tiene como objetivo la construcción, otra vez entre colegas, de un punto de vista compartido sobre la profesión, basado en las realidades de cada uno. Por lo tanto, se trata de tener una objetividad para construir un primer mundo común, el del mundo profesional. Aquí los protagonistas, todavía reunidos en grupos 'profesión', intentaron formalizar con palabras, de manera argumentada (sin necesariamente ser consensual) sus puntos de vista sobre su trabajo en relación con las exigencias y restricciones. Esto ha permitido a estos colegas de interrogar sus maneras de trabajar y ampliar su visión de los medios desplegados ante la organización formal. Estas dos primeras fases de la GRT permiten la formalización de una primera forma de la evaluación, la que está realizada por estos colegas de trabajo.

La tercera fase, la tercera intención, tiene como objetivo la transición de un enfoque específico a un enfoque integral para entender el trabajo en equipo, un mundo común más amplio. Si se trataba de producir conocimientos, esta vez queríamos entender la ' fabricación' del trabajo social de una manera ampliada, a través de la comunicación indirecta. Los cuatro grupos, a través de esta metodología de intervención, comunicaron y compartieron preguntas y respuestas. Había una jerarquía estatutaria entre los participantes, entre los grupos. Porque queríamos analizar el trabajo del ' equipo de las intervenciones sociales', esta programación organizativa nos ha obligado a encontrar soluciones que permitan una comunicación entre los grupos sin interrumpir la expresión. Tomamos la idea de escenificar varios grupos de profesiones diferentes del sociopsicoanálisis. Jean Luc Prades explica que la multiplicidad de grupos, cuando se reúnen, recomponen la institución, permitiendo a todos trascender la primera identidad para una comprensión del acto entero de la institución (Prades, 2007). Esta última fase permitió la elaboración de otra forma evaluativa, la del trabajo percibido por los demás. 
La experiencia que se acumula (Oddone, Re \& Briante 1981) en los grupos homogéneos en primer lugar, entre los grupos en segundo lugar, nos permitió obtener nuevos elementos sobre el trabajo. Esta modalidad de producción de conocimientos ha combinado la expresión y validación iniciando una progresión entre subjetividad y objetividad, entre colegas y entre miembros de un mismo equipo. La validación de las crónicas de actividad creadas a partir de las observaciones de situaciones de trabajo, de las síntesis, de las preguntas y respuestas de cada grupo, fueron etapas necesarias para la construcción de un juicio científico que ha dado un estatuto particular a los trabajadores: ser experto de su trabajo. Estos expertos han formulado conocimientos esperando el reconocimiento conceptual, conocimientos que no pertenecen a ninguna disciplina porque se concibieron a través de su experiencia. La validación entre varios permitió su conceptualización.

A partir de estos dos relatos, construimos la evaluación final. Analizamos, un informe de los conocimientos obtenidos del trabajo real por escrito y lo transmitimos a la dirección general. Este trabajo colectivo dió a conocer un punto de vista cualitativo de lo que produce la organización del trabajo, un punto de vista científico que se hace conmensurable a los otros puntos de vista.

\section{La evaluación ergológica al servicio de un ergo- management}

Ahora proponemos examinar el interés de la evaluación ergológica para las instituciones públicas.

Nuestro trabajo muestra que la evaluación ergológica ha sido beneficiosa para los trabajadores movilizados en los GRT porque se han apropiado de una especie de legitimidad para hablar de su propio trabajo y de la organización. Después, quisieron y solicitaron la versión escrita de su evaluación para mostrar sus conocimientos y justificar sus posiciones contra la organización prescrita. Así, la evaluación ergológica ha hecho posible poner de relieve otro tipo de valor, naturalmente presente en la empresa, el valor apropiado por cada uno en el proceso de producción. Y lo sabemos, los trabajadores necesitan ser valorados y reconocidos en y por lo que hacen, especialmente porque, a menudo saben mejor para sugerir maneras de mejorar la eficiencia (Hirigoyen, 2017). Sin embargo, la apropiación de los 'conocimientos de experiencias' por los agentes de dirección ha sido difícil, porque esos líderes no confiaron en nuestro dispositivo. Esas resistencias implican reservas para el valor y la utilidad de los conocimientos emergentes y esto va en contra de la búsqueda de la eficacia en el trabajo y más especialmente aquí, de la eficacia del trabajo efectuado para responder a una misión de servicio público.

La evaluación ergológica pretende que los conocimientos producidos por las evaluaciones cualitativas o cuantitativas alcancen el mismo nivel de cientificidad; su intención es lograr que la cientificidad de los nuevos conocimientos sea posible gracias a la concepción y concientización individual, colectiva y social, por los trabajadores, en su actividad de trabajo; por estas razones dicha evaluación ergológica permite que cada punto de vista sea importante para la organización del trabajo. Más allá de las ambiciones informativas de una evaluación clásica, la evaluación ergológica es también un instrumento democrático, solo si se considera que el diálogo es el fundamento de la democracia y que la democracia nace del Hombre $\left.{ }^{6}\right]$, de su actividad. Más que un 
instrumento de gestión, es un instrumento para los administradores. La evaluación ergológica es también un instrumento para todos los trabajadores, cualquiera sea su posición en el organigrama.

Cuando la empresa permite la producción de conocimientos del trabajo, así como también la complementariedad de los puntos de vista, la evaluación ergológica asume también un papel político. Nuestro trabajo consiste en ver, en particular, las consecuencias de un enfoque excesivamente agresivo de la lógica mercantil sobre la organización de actividades que están en el centro de un proyecto político (en el sentido de politeia) como lo es el trabajo social, así como también la salud o la enseñanza.

Las lógicas mercantiles transforman las profesiones, debilitan las identidades profesionales, debilitan los cuerpos, desmantelan los colectivos, neutralizan las relaciones e interacciones. Cambian el sentido del trabajo, interrogan sus objetivos y ponen en duda lo que se espera de cada uno a todos los niveles del organigrama. La evaluación ergológica plantea el vivir juntos como un problema común y plantea también la necesidad de pensar, con varias voces lo que significa trabajar al servicio del público, trabajar juntos para producir la riqueza esencial para construir y mejorar el mundo común en el que vivimos luchando contra las disparidades y la injusticia. Más que un gasto, estas actividades de servicio público son inversiones sociales. Es por esta razón que hacen obligatorio el debate colectivo y la articulación entre lo político, lo mercantil, la organización y lo profesional, sabiendo que cada uno de estos sectores cumple objetivos específicos y tienen temporalidades específicas. Estas actividades requieren que el suplemento de valor generado a través de la implicación individual en el trabajo general sea considerado y reconocido, porque sin esta implicación individual, no existirían.

Si la evaluación es aceptar riesgos porque puede revelar posibles distancias entre lo que se creía que se hacía y lo que realmente se hace, la evaluación ergológica requiere de la presencia de otros. Dicha evaluación cambia los principios normalmente establecidos de la organización del trabajo. Ya no hay por un lado los que saben y por otro los que ignoran, los que saben sabiendo para los que ignoran (Schwartz, 2012). La instrucción mutua admite una circularidad de conocimientos que se opone a una concepción descendente. Permite regenerar las directivas, revisar las lógicas a partir del trabajo real, del trabajo efectivamente realizado. Ofrece la posibilidad de gobernar más de cerca las realidades del trabajo y la de sus destinatarios. La evaluación ergológica es por lo tanto, una ayuda para quién quisiera actuar como ergo-manager [ $\left.{ }^{7}\right]$ El ergomanagement es un proyecto: el de una nueva gestión que quiere ser más democrática y por lo tanto menos traumática. Una gestión que no se basa en un conocimiento hegemónico, autocrático que no admite la prevalencia del poder sobre los conocimientos, que no niega la realidad del trabajo. Una gestión del trabajo por el trabajo, que autoriza los debates, la confianza, que también interesa a todos en el propósito del trabajo.

Es posible concluir con el postulado de que el ergo-manager es por lo tanto quién acepta, y permite que la complementariedad de las evaluaciones que solicita, sean retorno de experiencias de las conceptualizaciones organizacionales y de 1 manageriales. Es el dirigente que reconoce que su actividad se inscribe en una combinación de actividades que le hace falta conocer y entender. Es un manager quién se arriesga a hacer de forma distinta y no convencional. De hecho, si las técnicas de 
gestión se centran hoy más que antes en la personna que trabaja, hoy como ayer, el sufrimiento laboral, el poder y el control marcan la vida cotidiana de trabajadoras y trabajadores. El ergo-management se distingue de ello indudableñente.

\section{BIBLIOGRAFÍA}

Bocquet, B. (2016). La fièvre de l'évaluation. Quels symptômes? Quels Traitements ? Villeneuve d'Ascq: Presses Universitaires du Septentrion.

Di Ruzza R \& Halevi J. (2003). De l'économie politique à l'ergologie. Lettre aux amis. Paris : Editions L'Harmattan.

Durrive L. (2015). L'expérience de normes. Comprendre l'activité humaine avec la démarche ergologique. Toulouse : Editions Octarès.

Gori, R. (2015). La fabrique des imposteurs. Lonrai. Actes sud Editions.

Hirigoyen M.F. (2017). Le harcèlement moral au travail. Paris : Presses Universitaires de France.

Janover L. \& Rubel M. (2003). Karl Marx, Philosophie. Saint Amand : Galimard.

Mendel G. \& Prades J.L. (2002). Les méthodes de l'intervention psychosociologique. Paris : Editions La Découverte.

Oddone I., Re, A., \& Briante G. (1981). Redécouvrir l'expérience ouvrière. Paris : Editions sociales.

Prades J.L et les membres de l'ADRAP. (2007). Intervention participative et travail social. Un dispositif institutionnel pour le changement. Paris : L'Harmattan.

Schwartz Y. (2000). Le paradigme ergologique ou un métier de philosophe. Toulouse : Editions Octarès.

Schwartz Y. \& Durrive L. (2009a). Travail et ergologie : entretiens sur l'activité humaine. Toulouse :

Editions Octarès.

Schwartz Y. \& Durrive L. (2009b). L'activité en dialogues : entretiens sur l'activité humaine. Toulouse : Editions Octarès.

Schwartz Y. (2012). Expérience et connaissance du travail. Millau : Les éditions sociales.

Worms F. (2012). Soin et politique. Paris : Presses Universitaires de France.

\section{NOTAS}

1. Este artículo presenta, de manera sintética, algunos elementos presentados en mi tesis doctoral de filosofía.

2. La investigación se llevó a cabo en Francia gracias a una Convención de formación en investigación industrial (Cifre) en la Caja de Subsidios Familiares del Departamento de Bouchesdu-Rhône (CAF - Fondo de Asignaciones Familiares en Francia). El propósito de este dispositivo es cofinanciar la formación de un estudiante en doctorado, contratado por una empresa francesa con una misión de investigación que le permite preparar su tesis doctoral. 
3. Que 'es en cierto sentido más rico que el único análisis del trabajo ; Porque se interesa en todos los 'usos de uno mismo', trascendiendo todos los límites sociales, temporales e institucionales; capaz de pensar las circulaciones y la 're-inversión' entre ellos. El análisis del trabajo asalariado aparece desde este punto de vista más pobre, en la medida en que sólo se trata de ciertos segmentos de la vida humana y o, la heterodeterminación de las normas (distintas según las épocas, o según las formas de gobierno del trabajo) restringe la esfera de las conquistas inventivas' (Schwartz, 2000, p. 422, traducción libre).

4. 'Los conocimientos académicos y los conocimientos no doctos tienden a ser conmensurablesmensurables y el diálogo que debe establecerse entre ellos es, por lo tanto, un 'diálogo igualitario' (este puede ser el significado que debe darse al 'diálogo socrático bidireccional')' (Di Ruzza \& Halevi, 2003, p. 68, traducción libre).

5. Desarrollado por Gérard Mendel, 'el sociopsicoánalisis trata de entender cómo el hecho social influye en el hecho psíquico individual, incluyendo el inconsciente. La práctica colectiva estudia cómo los actores, organizados en grupos específicos (grupos homogéneos de profesiones) y en el marco de su actividad diaria, analizan ellos mismos las fuerzas que influyen en su personalidad' (Mendel \& Prades, 2002, p. 53, traducción libre).

6. '(...) mientras que Hegel piensa que el estado es la base, y el hombre es el estado cambiado en individuo, la democracia nace del hombre y considera que el estado es el hombre cambiado en objeto' ; es 'el hombre socializado' (cita de Karl Marx in Janover \& Rubel, 2003, introducción, traducción libre).

7. 'El ergo-manager significa que, en todos los niveles de las organizaciones humanas, dejamos de negar las normas anteriores, cosificando los socios, interlocutores, ejecutor, usuarios..., como si no fueran personas, (...). Esto implica una inquietud, una vigilancia, una preocupación permanente para aquellos que tienen esta responsabilidad o este poder, en su relación con los trabajadores' (Schwartz \& Durrive, 2009a, p. 242, traducción libre). Esta postura es pertinente para todos los niveles jerárquicos.

\section{AUTORES}

\section{INGRID FOUCHECOURT-DROMARD}

Aix Marseille Univ, CNRS, Centre Gilles Gaston Granger (UMR 7304), Aix-en-Provence, France ingdrom@hotmail.fr 